\title{
Review of: "ELMO1 signaling is a promoter of osteoclast function and bone loss"
}

\author{
Christel Verollet ${ }^{1}$, Brigitte Raynaud $^{1}$ \\ 1 Institute of Pharmacology and Structural Biology
}

Potential competing interests: The author(s) declared that no potential competing interests exist.

Bone undergoes continual remodeling by sequential action of bone-resorbing osteoclasts (OCS) and boneforming osteoblasts. Osteoblasts develop from cells of mesenchymal origin while OCs differentiate trough fusion of myeloid precursors under the control of the key osteoclastogenic cytokine, receptor activator of NF-KB ligand and macrophage colony stimulating factor. Excessive bone resorption results in osteoporosis, characterized by reduced bone density, alteration of bone structure, pain and increased risk of fracture. In addition, diseases such as rheumatoid arthritis present inflammatory synovitis associated with OCdependent bone destruction. Most clinically approved anti-resorption drugs enhance bone mass by either inducing OC apoptosis or impairing OC differentiation, but they also affect bone formation. The reason is that OCs can indirectly stimulate bone formation, independently of bone resorption, in particular by secreting proteins such as clastokines ${ }^{1}$. While prevalence of osteoporosis and OC-dependent diseases dramatically increases worldwide, it is necessary to identify alternative therapeutic approaches that target the activity of OCs while preserving their pool. To date, very few targets meeting these criteria are available. The first tempting target has been cathepsin K, a key OC protease. Indeed, cathepsin K inhibitors are now available and proved to be efficient as anti-resorptive molecules preventing pathological bone loss but adverse effects have been reported ${ }^{2-4}$. Another promising strategy is to target the OC specific adhesive actin structure (the sealing zone) by impacting the b3 integrin, the major OC integrin, or the exchange factor Dock5, a guanine nucleotide exchange factor of the small GTPase Rac which controls actin dynamics inside the sealing zone ${ }^{5-7}$. Inhibitors of these two targets are still in the preclinical or early clinical stage. In this context, the paper from Aranjelovic and $\mathrm{l}^{8}$ recently identified ELMO1, that is part of a complex with Dock5 and Rac1 ${ }^{9}$, as another potential target to control OC function without significantly affecting their number.

ELMO1 protein was originally identified as an activator of the small Rac1-GTPase that controls actin polymerization, and a key player in efferocytosis and cell migration ${ }^{10}$. The Rho-GTPases family (Racl and Rac2) has been reported to perform multiple roles during OC differentiation, including essential dynamic rearrangement of the actin cytoskeleton (for review see ${ }^{11,12}$ ). However, the exact role of ELMO1 in the osteclastogenesis was not elucidated before this study.

In Aranjelovic and al. $^{8}$, the authors obtained the first indication of a role of ELMO1 in the osteoclastogenesis process from the analysis of available databases that allowed a correlation between 
single-nucleotide polymorphisms in ELMO1 with various bone or joint diseases. Interestingly, Elmo1 +/- or Elmo1-/- mice do not have bone defects nor strong effects on OC number or osteoblast mineralization at the steady-state. However, the role of ELMO1 is revealed in pathological conditions. Indeed, this study elegantly evaluated the phenotype of Elmo1 +/- or Elmo1-/- mice in various and complementary in vivo mouse models of osteoporosis/bone erosion as well as arthritis, using morphological analysis (whole body DEXA scan and trabecular bone microCT) and quantification of OC markers. In particular, they explored two classical models of osteoporosis (osteoprotogerin-deficient mice and in ovariectomized mice mimicking post-menauposal osteoroporosis), and two independent models of arthritis (collagen- or K/BxN serum induced- arthritis). In all of these cases, the loss of ELMO1 significantly counteracted bone erosion. They also confirmed their results with Elmo ${ }^{\mathrm{fl} / \mathrm{fl}} \mathrm{C} \times 3 \mathrm{cr} 1$-Cre mice which is supposed to delete ELMO1 specifically in OCs, or at least in an OC sub-population ${ }^{13}$. Furthermore, compared to control mice, OCs differentiated from bone marrow precursors derived from ELMO1-deficient mice display a strong defect in their bone resorption activity while their number and their multinucleation status were not modified. These data suggest that ELMO1 is important for OC function but not for OC differentiation. Indeed, osteoclastic differentiation does not appear to be significantly affected by ELMO1 loss, although the expression of a classical marker of OC maturation, the b3 integrin, increased. These results were further confirmed using additional in vitro models, including CRISPR/Cas9 Elmo1 deletion in Hoxb8 OCs ${ }^{14}$. Most of these data on the positive role of ELMO1 in OC function both ex vivo and in vivo were published at the same time by the group of Yan J. ${ }^{15}$, while in their experiments they found that ELMO1 deletion also affects OC differentiation. In addition, Aranjelovic and al. ${ }^{8}$ showed that impaired bone resorption in ELMO1-deficient OCs was accompanied by disruption of the F-actin bone resorption apparatus of OCs (belts and sealing zones on glass and bone, respectively) in respect with their functional defect. They also identified several new ELMO1-dependent regulated genes involved in OC function, such as cathepsin G and myeloperoxidase, using complementary transcriptomics and proteomics approaches. They went further and provided evidence that this new candidate could be druggable. Indeed, based on the structure of ELMO1 with one of its interactors, they designed an inhibitory peptide. Importantly, this peptide was able to impair OCmediated bone resorption in vitro. While promising, many works remain in order to evaluate the consequences of ELMO1 inhibitory peptides in vivo at the steady state and in pathological conditions, but also their potential side effects. Indeed, ELMO1 is widely expressed and an upstream regulator of small Rac1-GTPase involved in several cellular functions in addition to OC function.

Taken together, these results showed the key role of ELMO1 as a signal regulator of OC function and not differentiation or number, making it an original new target. In addition, this important publication highlights new cellular mechanisms controlling OC bone resorption activity which may be exploited further towards innovative applications in the context of bone resorption disorders including age-related osteoporosis and arthritis. 

117, 1753-1756, doi:10.1002/jcb.25560 (2016).

2 Drake, M. T., Clarke, B. L., Oursler, M. J. \& Khosla, S. Cathepsin K Inhibitors for Osteoporosis: Biology, Potential Clinical Utility, and Lessons Learned. Endocr Rev 38, 325-350, doi:10.1210/er.2015-1114 (2017),

3 Kim, B., Cho, Y. J. \& Lim, W. Osteoporosis therapies and their mechanisms of action (Review). Exp Ther Med 22, 1379, doi:10.3892/etm.2021.10815 (2021).

4 Stone, J. A., McCrea, J. B., Witter, R., Zajic, S. \& Stoch, S. A. Clinical and translational pharmacology of the cathepsin K inhibitor odanacatib studied for osteoporosis. BrJ Clin Pharmacol 85, 1072-1083, doi:10.1111/bcp.13869 (2019).

5 Mounier, L. et al. Novel 2,7-Diazaspiro[4,4]nonane Derivatives to Inhibit Mouse and Human Osteoclast Activities and Prevent Bone Loss in Ovariectomized Mice without Affecting Bone Formation. J Med Chem 63, 13680-13694, doi:10.1021/acs.jmedchem.0c01201 (2020).

6 Murphy, M. G. et al. Effect of L-000845704, an alphaVbeta3 integrin antagonist, on markers of bone turnover and bone mineral density in postmenopausal osteoporotic women. J Clin Endocrinol Metab 90, 2022-2028, doi:10.1210/jc.2004-2126 (2005).

7 Vives, V. et al. Pharmacological inhibition of Dock5 prevents osteolysis by affecting osteoclast podosome organization while preserving bone formation. Nat Commun6, 6218, doi:10.1038/ncomms7218 (2015),

8 Arandjelovic, S. et al. ELMO1 signaling is a promoter of osteoclast function and bone loss. Nat Commun 12, 4974, doi:10.1038/s41467-021-25239-6 (2021).

9 Kukimoto-Niino, M. et al. Cryo-EM structure of the human ELMO1-DOCK5-Rac1 complex. Sci Adv 7, doi:10.1126/sciadv.abg3147 (2021).

10 Kukimoto-Niino, M., Ihara, K., Murayama, K. \& Shirouzu, M. Structural insights into the small GTPase specificity of the DOCK guanine nucleotide exchange factors. Curr Opin Struct Biol 71, 249-258, doi:10.1016/j.sbi.2021.08.001 (2021).

11 Gao, L., Kong, L. \& Zhao, Y. The Regulatory Role of Rho GTPases and their Substrates in Osteoclastogenesis. Curr Drug Targets22, 1064-1070, doi:10.2174/1389450121666200925150446 (2021). 12 Ory, S., Brazier, H., Pawlak, G. \& Blangy, A. Rho GTPases in osteoclasts: orchestrators of podosome arrangement. EurJ Cell Bio/87, 469-477, doi:10.1016/j.ejcb.2008.03.002 (2008).

13 Madel, M. B. et al. Dissecting the phenotypic and functional heterogeneity of mouse inflammatory osteoclasts by the expression of Cx3cr1. Elife 9, doi:10.7554/eLife.54493 (2020).

14 Di Ceglie, I. et al. Genetic modification of ER-Hoxb8 osteoclast precursors using CRISPR/Cas9 as a novel way to allow studies on osteoclast biology. J Leukoc Biol 101, 957-966, doi:10.1189/jlb.1AB0416- 
180RR (2017).

15 Liang, X. et al. ELMO1 Regulates RANKL-Stimulated Differentiation and Bone Resorption of Osteoclasts. Front Cell Dev Biol 9, 702916, doi:10.3389/fcell.2021.702916 (2021). 